\title{
Duodenal Sensory Neurons Project to Sphincter of Oddi Ganglia in Guinea Pig
}

\author{
Audra L. Kennedy and Gary M. Mawe \\ Department of Anatomy and Neurobiology, The University of Vermont, Burlington, Vermont, 05405
}

Retrograde labeling of duodenum-sphincter of Oddi (SO) preparations in vitro with the carbocyanine dye Dil revealed that duodenal neurons project to the SO. The duodenum-SOprojecting neurons were immunoreactive (IR) for choline acetyltransferase but not nitric oxide synthase or calretinin, indicating that this is a cholinergic projection and that this pathway is distinct from the circuitry involved in the ascending limb of the peristaltic reflex. Approximately $20 \%$ of the duodenum-SO projection neurons were IR for calbindin. Calbindin-IR nerves within SO ganglia degenerated when the SO was maintained in organ culture alone, but persisted when the SO was cultured with the duodenum intact. Therefore, SO ganglia are a target of the calbindin-positive duodenum-SO projection. Because calbindin is a marker of intrinsic sensory neurons that have pro-

One century ago, Bayliss and Starling (1899) demonstrated that peristaltic activity in the gut is performed by "the local nervous mechanism" and that basic excitatory and inhibitory reflex circuitry must be intrinsic to the enteric nervous system (ENS). In addition to the local neural networks underlying peristalsis, other peripheral neural circuits have been discovered that are likely to influence gut function by providing communications between distinct sets of ganglia and between the gut and accessory organs. These include neural projections from enteric myenteric neurons to the prevertebral sympathetic ganglia, the gallbladder, and the pancreas (Mawe, 1995). The aim of this study was to test the hypothesis that a neural link exists between the myenteric ganglia of the duodenum and the ganglia of the sphincter of Oddi (SO).

The SO is a smooth muscle sphincter, located at the terminal portion of the common bile duct, which serves to regulate the flow of bile and pancreatic juices into the duodenum. Although the exact mechanisms of SO function are not understood, it is likely that changes in SO tone involve neurohormonal inputs to the ganglionated plexus of the $\mathrm{SO}$ and a coordinated output from these ganglia. After a meal, SO tone is altered by the release of cholecystokinin (CCK) from the duodenal mucosa, which acts through a neural mechanism to decrease SO resistance (Behar and Biancani, 1987; Vogalis et al., 1989; Hanyu et al., 1990).

Several lines of evidence suggest that this neural mechanism may involve a projection to SO ganglia from CCK-sensitive duodenal sensory neurons: (1) duodenal myenteric neurons of the

Received April 27, 1998; revised July 16, 1998; accepted July 21, 1998.

This work was supported by National Institutes of Health Grants NS26995 and DK45410. We thank Drs. Rodney Parsons and Kirk Hillsley for valuable discussion, and Mr. Jason Hemming for technical assistance.

Correspondence should be addressed to Dr. Gary M. Mawe, Department of Anatomy and Neurobiology, C-423 Given Building, The University of Vermont, Burlington, VT 05405.

Copyright (ㄷ) 1998 Society for Neuroscience $\quad 0270-6474 / 98 / 188065-09 \$ 05.00 / 0$ cesses that pass to the mucosa, these neurons are in position to detect the release of a compound from the mucosa and signal its release to SO ganglia. When applied to retrogradely labeled neurons, cholecystokinin (CCK) elicited a prolonged depolarization, indicating that duodenum-SO-projecting neurons could be capable of detecting CCK released from the mucosa. It is proposed that the role of the intrinsic sensory neurons that project to the SO may be to signal the postprandial release of CCK, thus providing an instruction to decrease $\mathrm{SO}$ resistance and facilitate the flow of bile into the duodenum.

Key words: enteric nervous system; myenteric plexus; calbindin; sphincter of Oddi; duodenum; sensory neurons; cholecystokinin

Australian possum are retrogradely labeled after injection of tracers into the SO (Padbury et al., 1993); (2) duodenal distension results in tetrodotoxin-sensitive changes in SO tone in the Australian possum (Saccone et al., 1994); (3) SO ganglia in the guinea pig are richly innervated by calbindin-immunoreactive (IR) nerve fibers, and calbindin-IR neurons are numerous in adjacent duodenal myenteric ganglia (Wells and Mawe, 1993); (4) type 2/AH cells in the myenteric plexuses of the guinea pig ileum (Nemeth et al., 1985; Schutte et al., 1997) and duodenum (Mutabagani et al., 1993), which are likely sensory neurons of the myenteric plexus (Kunze et al., 1995), are responsive to CCK; and (5) although SO neurons respond to $\mathrm{CCK}$, the efficacious concentration is orders of magnitude greater than the serum levels of CCK after a meal (Gokin et al., 1997).

Experiments were designed to identify the neurons in the duodenum that project to the SO and to characterize these projection neurons on the basis of their chemical coding patterns. These experiments were devised in part on existing knowledge of the guinea pig enteric nervous system. Guinea pig myenteric neurons that are immunoreactive for the calcium-binding protein calbindin D28 are intrinsic primary sensory neurons (Costa et al., 1992; Song et al., 1994; Kunze et al., 1995). Furthermore, these neurons have projections to the intestinal villi (Song et al., 1994). Because calbindin-IR nerve fibers are abundant in SO ganglia, but calbindin-IR cell bodies are rare, it was tested whether the duodenum-SO-projecting neurons constitute this calbindin fiber immunoreactivity. To test whether duodenum-SO-projecting neurons are capable of sensing CCK, we recorded from retrogradely labeled neurons with intracellular microelectrodes and applied CCK to determine their responsiveness to the peptide.

\section{MATERIALS AND METHODS}

Preparation of tissue. Adult guinea pigs of either sex weighing 200-350 gm were deeply anesthetized with halothane and exsanguinated. This 
method has been reviewed and approved by the Institutional Animal Care and Use Committee of the University of Vermont (protocol 95056). Sterile instruments, dishes, glassware, and Krebs' solution were used in all the experiments involving whole-mount culture. After tissue removal, the duodenum was opened with a longitudinal incision on the side opposite the common bile duct. The tissues were pinned, mucosal side up, in a Sylgard-lined dish in a solution of iced Krebs' solution, and the mucosal and submucosal layers were removed. The Krebs' solution, aerated with $95 \% \mathrm{O}_{2}$ and $5 \% \mathrm{CO}_{2}$, contained in (mM): $\mathrm{NaCl}, 121 ; \mathrm{KCl}$, 5.9; $\mathrm{CaCl}_{2}, 2.5 ; \mathrm{MgCl}_{2}, 1.2 ; \mathrm{NaHCO}_{3}, 25 ; \mathrm{NaH}_{2} \mathrm{PO}_{4}, 1.2$; and glucose, 8. Nifedipine $(5 \mu \mathrm{M})$ was added to minimize muscle contractions. The preparations were thoroughly rinsed throughout the dissection and transferred to a second sterile Sylgard-lined Petri dish before culture.

Retrograde labeling with DiI. Retrograde-labeling studies were performed using techniques similar to those developed and previously described by Brookes et al. (1991a,b). Briefly, small glass beads (200-300 $\mu \mathrm{m}$; Sigma, St. Louis, MO) were coated with a $1 \mathrm{~mm}$ solution of the dialkylcarbocyanine probe DiI (1,1'-didodecyl-3,3,3',3'-tetramethylindocarbocyanine perchlorate; Molecular Probes, Eugene, OR) in 100\% ethanol. Using fine forceps, DiI-labeled beads were placed within the SO region and gently but firmly pressed onto the tissue. The border of the SO could be visualized as a very discernible ridge of thickened tissue under low levels of magnification $(6.5 \times-16 \times)$ with a dissecting microscope. Although the SO border could be visualized under bright-field microscopy, a very fine-tipped permanent marker was used to delineate the border of the SO on the glass slides containing the whole-mount preparations for more accurate visualization under fluorescence microscopy. This method of DiI application enables the introduction of a high concentration of DiI to a small, restricted site. After the desired culture period $(48-72 \mathrm{hr})$, the tissue was fixed with $2 \%$ formaldehyde from paraformaldehyde containing $0.2 \%$ picric acid, mounted with $50 \%$ glycerol and 50\% $0.1 \mathrm{M}$ PBS, and visualized with fluorescence optics using a rhodamine filter set.

Organotypic culture techniques. Dissections for all organotypic culture studies were performed in a horizontal laminar flow hood with sterile solutions, instruments, and dishes. After dissection, Krebs' solution was replaced with culture medium consisting of DMEM and F-12 containing $10 \%$ horse serum, gentamycin $(10 \mathrm{mg} / 100 \mathrm{ml})$, amphotericin $\mathrm{B}(12.5$ $\mu \mathrm{g} / 100 \mathrm{ml})$, nifedipine $(1 \mu \mathrm{M})$, and antibiotic-antimycotic solution (1 $\mathrm{ml} / 100 \mathrm{ml}$; all from Sigma). The preparations were placed on a slowly rocking shaker table inside a $37^{\circ} \mathrm{C}, 95 \% \mathrm{O}_{2}$ and $5 \% \mathrm{CO}_{2}$ incubator. The culture medium was replaced every $24 \mathrm{hr}$. To determine the optimal time in culture for transport of the dye, preparations were maintained in organotypic culture for either 48 or $72 \mathrm{hr}$. No noticeable differences were observed between the two time periods, and, therefore, the $48 \mathrm{hr}$ time period was used in most retrograde-labeling experiments.

Immunohistochemical studies. Immunohistochemical studies performed in these experiments were similar to those previously described for the SO (Wells et al., 1995). Tissues were fixed in a solution of $2 \%$ paraformaldehyde and $0.2 \%$ picric acid in $0.1 \mathrm{~m} \mathrm{PBS}, \mathrm{pH} 7.4$, for $2-24 \mathrm{hr}$, after which circular muscle was removed from the preparations.

For studies combining DiI labeling with immunohistochemistry, the tissue was permeabilized in 50\% glycerol and 50\% PBS for $15 \mathrm{~min}, 80 \%$ glycerol and 20\% PBS for $15 \mathrm{~min}$, and $100 \%$ glycerol for $60 \mathrm{~min}$, as described by Brookes et al. (1991a,b) and Song et al. (1992). Glycerol was used instead of Triton X-100, which causes leakage of DiI from retrogradely labeled cells. After permeabilization, the tissue was treated with the appropriate antibodies (see below).

For immunohistochemical studies not involving DiI-labeled tissue, the whole-mount preparations were incubated in primary antisera that were diluted in PBS $(0.1 \mathrm{M})$ containing the detergent Triton X-100 $(0.5 \%)$ to enhance permeabilization. The preparations remained in the primary antiserum for $24 \mathrm{hr}$ at room temperature or for $48 \mathrm{hr}$ at $4^{\circ} \mathrm{C}$. After thorough rinsing, the preparations were exposed to species-specific secondary antibodies labeled with fluorophores. For the concurrent detection of two antigens in the same preparation, primary antibodies raised in two different species were combined with species-specific secondary antibodies conjugated to contrasting fluorophores. Standard immunohistochemistry control experiments, including absorption of primary antisera with antigen and testing secondary antisera in the absence of primary antisera, were conducted.

In some cases, intense DiI fluorescence in retrogradely labeled neurons was detected with the FITC filter set, thus making it difficult to distinguish FITC immunofluorescence from retrogradely transported DiI in these preparations. Therefore, in all cases in which DiI tracing was combined with immunohistochemistry for a given antigen, experiments were done with both FITC-labeled secondary antisera and with the biotin-avidin peroxidase complex.

For studies combining DiI labeling with immunohistochemistry for ChAT, the tissue preparations were imaged using a laser-scanning confocal microscope (Noran Instruments, Oz System, Middleton, WI) mounted on a Nikon Diaphot inverted microscope using a $20-\mathrm{X}$ air objective. Using Noran's InterVision software, a z-series was collected in the interleave mode. For DiI imaging, the confocal microscope system was switched between an excitation wavelength of $568 \mathrm{~nm}$ and an emission wavelength of 585-630 nm. For ChAT-IR Alexa 488 imaging, the system was switched between an excitation wavelength of $488 \mathrm{~nm}$ and an emission wavelength of $515-550 \mathrm{~nm}$. A narrow bandpass barrier filter was used for collecting fluorescent images at both mentioned emission wavelengths. The acquired digital image TIFF files were transferred to a Macintosh G3 PowerPC computer, and images were processed using Adobe Photoshop LE (Adobe Systems, Mountain View, CA) and printed using a Codonics NP 1600 printer.

Antisera used. Mouse anti-calbindin D-28 antiserum was used at a dilution of 1:500 (Sigma); rabbit anti-calretinin antiserum was used at a dilution of 1:5000 (Chemicon International, Temecula, CA); rabbit antinitric oxide synthase (NOS) antiserum derived from brain NOS (Santa Cruz Laboratories, Santa Cruz, CA) was used at a dilution of 1:250; goat anti-ChAT antiserum was used at a dilution of 1:250 (Chemicon, Temecula, CA). Secondary antisera used in all experiments (Jackson ImmunoResearch, West Grove, PA) were conjugated to fluorescein isothiocyanate (FITC; $1: 100)$, indocarbocyanine (Cy3; 1:500), or biotin $(1: 400)$. When biotin-conjugated antisera were used, streptavidin Alexa 488 (1: 250; Molecular Probes, Eugene, OR) or streptavidin peroxidase (1:200) was applied to the tissue, and preparations were processed with diaminobenzidine reagent set purchased from Kirkegaard \& Perry Gathersburg, MD).

Analysis of DiI-labeled preparations. Whole-mount preparations of duodenal myenteric plexus and SO ganglionated plexus were analyzed to establish the distribution of nerve cell bodies in the myenteric plexus after DiI application to the SO. Preparations were viewed under a Zeiss fluorescence photomicroscope equipped with an HBO $100 \mathrm{~W}$ mercury arc lamp. A $565 \mathrm{~nm}$ primary filter-590 nm secondary filter combination was used to visualize DiI and Cy3. A $485 \mathrm{~nm}$ primary filter-520 nm secondary filter combination was used to visualize FITC. Hardware for computerized mapping of each preparation included a motorized $x-y$ stage attached to a Zeiss fluorescent photomicroscope equipped with an HBO $100 \mathrm{~W}$ mercury arc lamp light source, Lucivid video hardware, and computer equipped with Windows 95. In each preparation, the exact location and chemical identity of each retrogradely labeled neuron could be recorded with a precision of $5 \mu \mathrm{m}$ using the computerized stage mapping system and Neurolucida software (MicroBrightfield, Colchester, VT). Neurolucida is a Microsoft Windows-based program that enables the digitizing of information from serial regions of microscopic images into a resulting map of the entire whole-mount preparation.

Electrophysiological recording and application with CCK. Tissue was cultured with DiI as described above. After $48-72 \mathrm{hr}$ in culture, the tissue was pinned out in a low volume $(2.5 \mathrm{ml})$ recording chamber and continuously bathed $(10 \mathrm{ml} / \mathrm{min})$ in aerated $\left(95 \% \mathrm{O}_{2}\right.$ and $\left.5 \% \mathrm{CO}_{2}\right)$ Krebs' solution at $33-35^{\circ} \mathrm{C}$. DiI-labeled cells were visualized using an inverted microscope (Nikon Diaphot) equipped with a $100 \mathrm{~W}$ UV light source and rhodamine filter cube. Glass microelectrodes used for intracellular recording from DiI-labeled cells in the duodenum were back-filled with neurobiotin $(2 \%$ solution in $1 \mathrm{M} \mathrm{KCl})$, and shanks were filled with $2 \mathrm{M}$ $\mathrm{KCl}$ and had resistances in the range of 80-120 M $\Omega$. An Axoclamp 2A amplifier with bridge circuitry for injecting positive and negative current pulses was used to record membrane potentials. CCK was applied by pressure ejection from glass micropipettes $(0.1 \mathrm{~mm}$ in Krebs' solution; $15-20 \mu \mathrm{M}$ tip diameter) by pulses of nitrogen gas $\left(300 \mathrm{~kg} / \mathrm{cm}^{2} ; 0.5-2 \mathrm{sec}\right.$ duration), and by bath application (100 nM).

\section{RESULTS}

\section{Retrograde labeling of duodenal neurons from the SO}

The retrograde tracer DiI was applied to the SO to identify duodenal neurons that project to the SO. When evaluating the results of these experiments, the application site was carefully examined to determine the extent of DiI spread. The dye spread at the application site had to be confined to the SO in order for a 

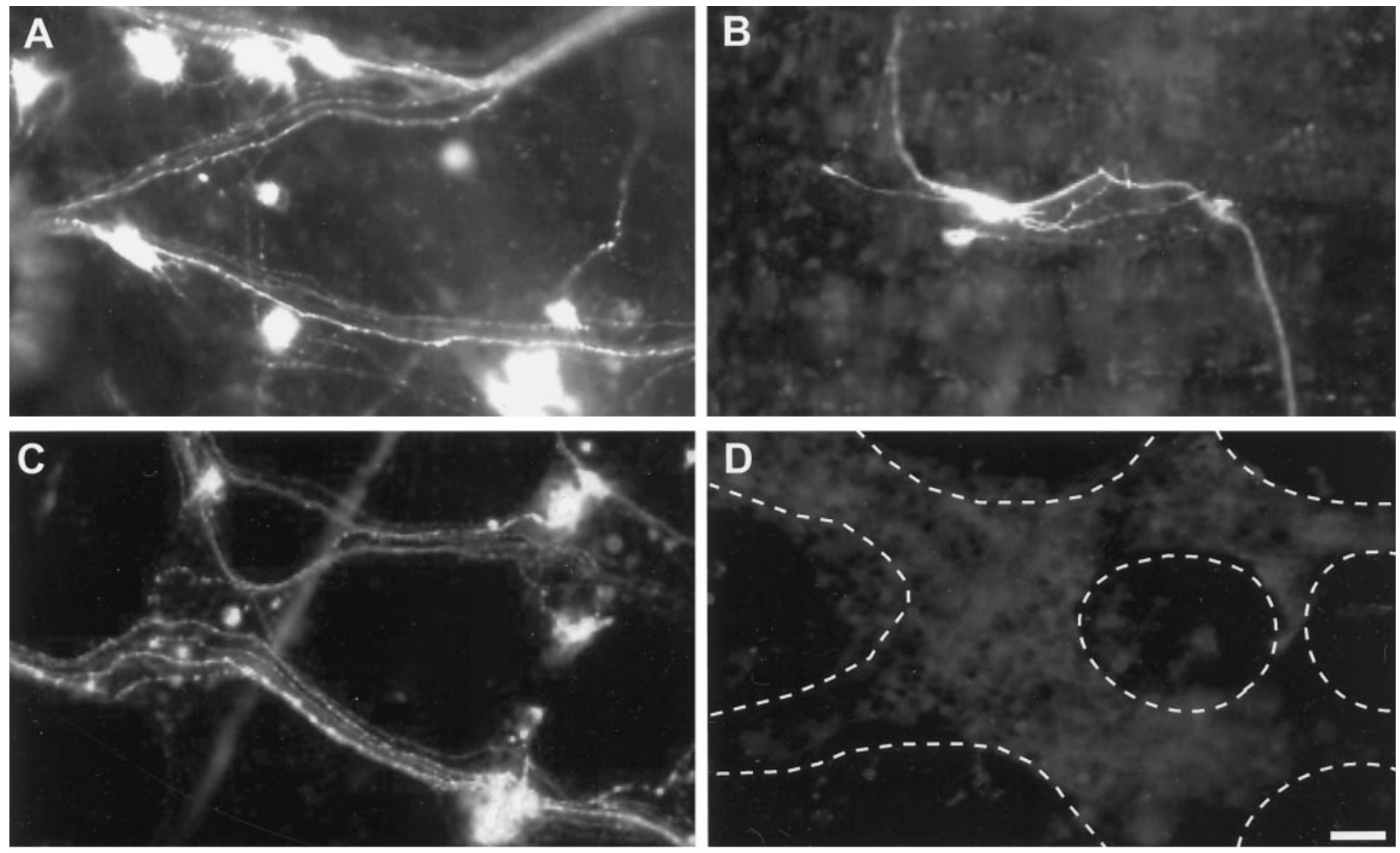

$\mathbf{E}$

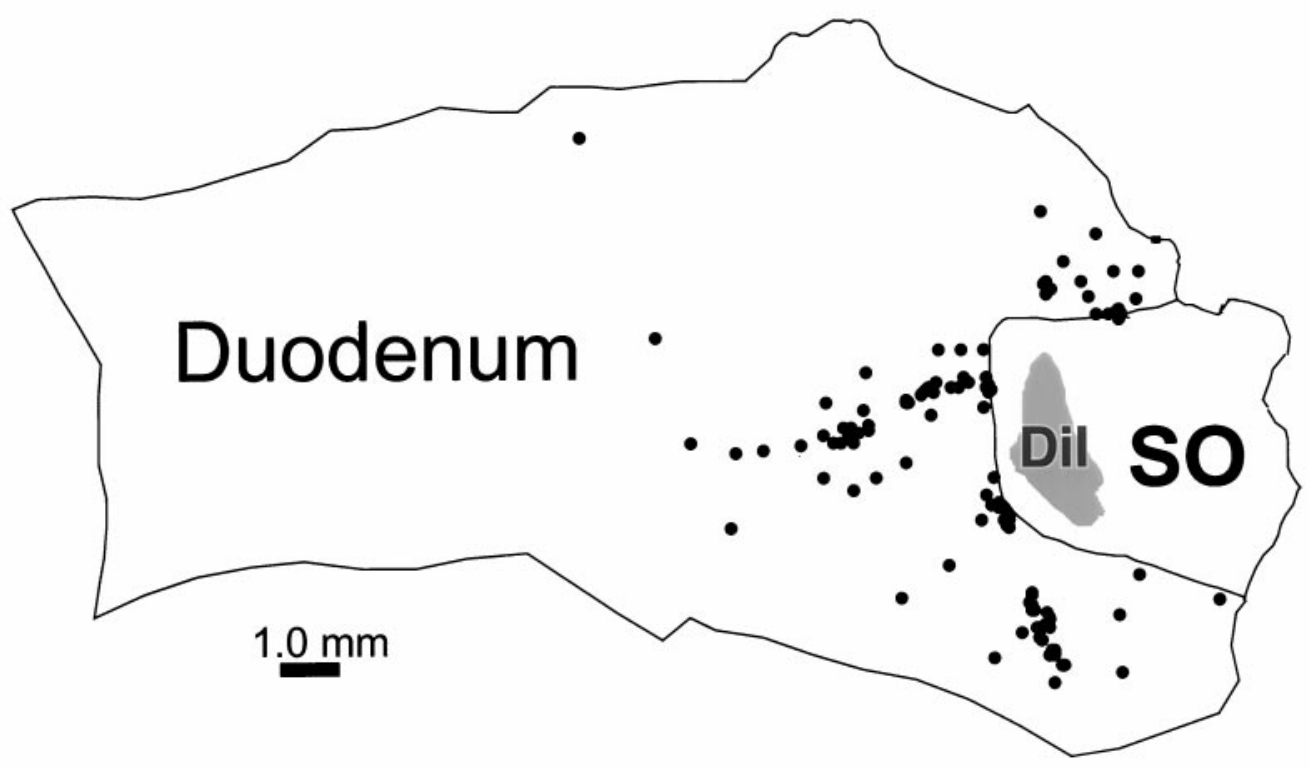

Figure 1. Neurons in the myenteric plexus of the duodenum are retrogradely labeled after application of the tracer DiI to the SO. $A-C$, Photomicrographs of duodenal myenteric ganglia that contain retrogradely labeled neurons after application of DiI to the SO and maintenance in organ culture for $48 \mathrm{hr} . D$, Photomicrograph of a duodenal ganglion near the SO from a preparation in which the SO-duodenal border was pinched with forceps before the application of DiI in the SO. No labeled neurons were observed in these preparations. $E$, A map generated with Neurolucida showing the outline of the preparation, the location of DiI application in the SO, and retrogradely labeled neurons in the duodenum $(n=121)$. In 10 preparations that were quantified, $116 \pm 20$ cells (range, 52-221) were retrogradely labeled in the myenteric plexus of the duodenum. Scale bar, $50 \mu \mathrm{m}$.

given preparation to be included in the study. The precise border of the SO could be visualized under low levels of magnification as a thickened ridge of tissue. This thickened area is apparent in Figure $5 D$ as a dark vertically running band of tissue indicated by the dotted line. A very fine-tipped permanent marker was used to delineate the SO border on glass microscope slides containing the whole-mount preparations for accurate identification of the border during analysis of the preparations.

DiI-labeled neurons were consistently observed in the duodenal myenteric plexus $48 \mathrm{hr}$ after application of DiI to the SO $(n=$ 40; Fig. $1 A-C$ ). Neurons were classified as DiI-positive if they possessed a bright, punctate labeling caused by the packaging of 
dye into vesicles and retrograde transport of vesicles from the origin of dye application to the cell bodies. Most often, the dendrites of these cells could also be visualized, and the cells could therefore be classified as Dogiel type I or type II based on their morphology. DiI-labeled axons could be followed along interganglionic fiber bundles that connected the ganglionated plexuses of the duodenum and the SO. Furthermore, there was a general pattern of labeling in which certain axonal pathways could be followed to the SO in all of the preparations.

Computer-generated maps of ten preparations were created using Neurolucida software, which included an outline of the tissue and the SO region as well as the application site (Fig. 1E). The creation of a computerized map enabled the precise localization of the distance and the coordinates of each neuron projecting to the $\mathrm{SO}$ and provided quantitative information regarding the contingent of duodenal neurons that project to the SO. In these preparations, $116 \pm 20$ cells (range, 52-221) were labeled with DiI in the myenteric plexus of the duodenum. The mean distance of these cells from the application site was $2821.9 \pm$ $208.2 \mu \mathrm{m}$. Although labeled neurons were observed as far as 12.7 $\mathrm{mm}$ from the SO border, $92 \%$ of the retrogradely labeled neurons were located within $5 \mathrm{~mm}$, and $50 \%$ of the DiI-labeled cells were located within $2.5 \mathrm{~mm}$ of the SO.

Control experiments were performed to confirm that DiIpositive neurons were labeled by retrograde axonal transport of DiI from the SO. In six preparations, the SO-duodenal junction was pinched with forceps before application of DiI to destroy axonal projections from the duodenum to the SO (Fig. $1 D$ ). It is likely duodenal neurons are labeled by active transport of DiI along the axons rather than by passive diff usion within the plasma membrane, because retrograde labeling occurs in a matter of days with active transport and requires weeks for passive transport (Honig and Hume, 1989). Furthermore, when DiI was applied to the $\mathrm{SO}$ in a previously fixed $\mathrm{SO}$-duodenum preparation, no labeled neurons were observed in the duodenum or the SO after $48 \mathrm{hr}$ in culture medium at $37^{\circ} \mathrm{C}$. Therefore, an additional set of control experiments was conducted to further verify that duodenal neurons were labeled from the dye application site. In these experiments, DiI was applied to the SO, and the preparations were organ cultured in the presence of colchicine, which causes a breakdown of microtubules and thus prevents axonal transport from occurring. With the disruption of axonal transport, by colchicine or by pinching the duodenal-SO border, no retrogradely labeled neurons were detected in the duodenum.

\section{Identification of the chemical coding of duodenum-SO projection neurons}

The myenteric plexus consists of several subtypes of neurons that express distinctive combinations of neuronal proteins, and serve distinctive functions. To determine which duodenal neurons project from the myenteric plexus to the SO, retrograde transport of DiI was combined with immunohistochemistry. The chemical coding markers evaluated were the biosynthetic enzymes, ChAT and NOS, and the calcium-binding proteins, calretinin and calbindin. The expression of these compounds does not appear to change within $48 \mathrm{hr}$ in organ culture, because the proportions of ChAT-, NOS-, calretinin-, or calbindin-labeled neurons in the myenteric plexus of the small intestine were not significantly different in control versus cultured preparations (J. Hemming, A. Kennedy, and G. Mawe, unpublished observations).
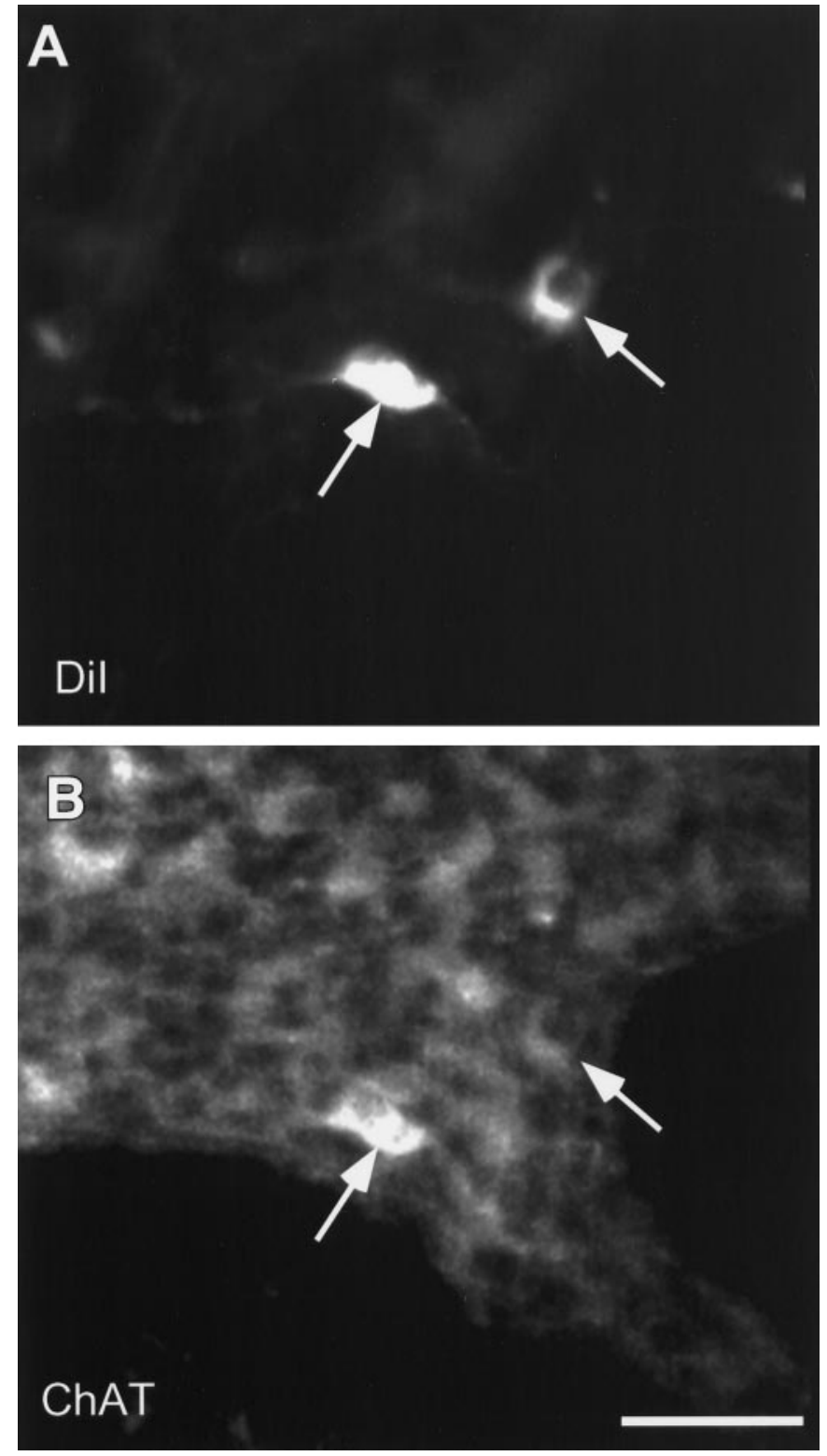

Figure 2. Neurons that are retrogradely labeled with DiI are immunoreactive for ChAT. $A$ and $B$ are confocal images of the same field demonstrating $\operatorname{DiI}(A)$ and ChAT immunoreactivity fluorescently labeled with streptavidin Alexa $488(B)$. Arrows indicate the positions of DiIlabeled neurons in $A$ and $B$. Scale bar, $50 \mu \mathrm{m}$.

\section{Duodenum-SO projection neurons are ChAT-positive, NOS-negative, and calretinin-negative}

A recent study of ChAT and NOS immunoreactivities in the duodenum has established that the vast majority of neurons in the duodenal myenteric plexus of the guinea pig are immunoreactive for either ChAT or NOS, but not both, enzymes (Clerc et al., 1998a,b). In the four preparations examined, the majority of DiI-labeled neurons could be clearly identified as ChAT-IR (Fig. 2). These DiI-positive neurons were intensely IR for ChAT, with clear borders and nuclear halos. The remainder of neurons appeared to be ChAT-IR, but immunoreactivity was more difficult to assess because clear outlines of the cells could not be distinguished. ChAT-IR neurons are often clustered in enteric ganglia, and it can be difficult to discern individual neurons. Consistent with the finding that DiI-labeled neurons were ChAT-positive, no 

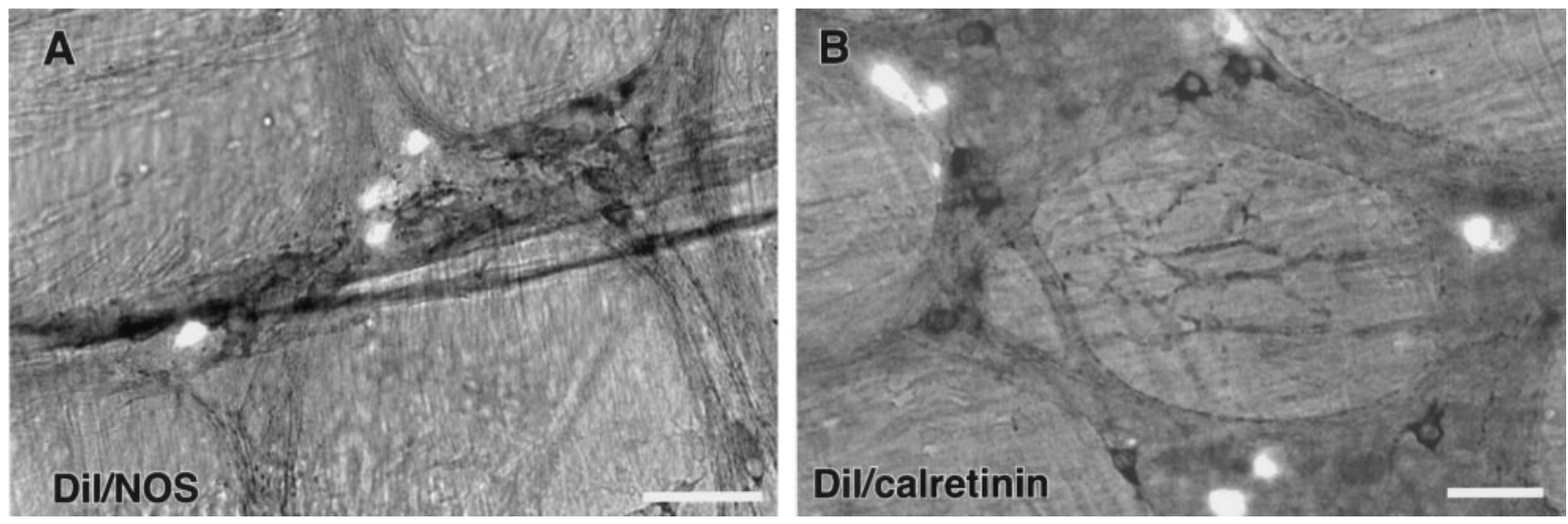

Figure 3. Neurons that project to the SO from the duodenum are neither calretinin- nor NOS-immunoreactive. $A$, Photomicrograph simultaneously demonstrating DiI (fluorescent cells) and NOS immunoreactivity-labeled with DAB (dark cells). The two groups are distinct populations of neurons. $B$, Photomicrograph simultaneously demonstrating DiI (fluorescent cells) and calretinin immunoreactivity-labeled with DAB (dark cells). Again, the two groups can be seen as distinct populations of neurons. Scale bar, $50 \mu \mathrm{m}$.

NOS-positive-DiI-labeled neurons were identified in the six preparations that were immunostained for NOS (Fig. $3 A$ ).

In the guinea pig small intestine and duodenum, calretinin is expressed by a subset of motor neurons and by interneurons that project in an ascending, or anal to oral, direction (Brookes et al., 1991a; Clerc et al., 1998b). Because the axons of many duodenumSO-projecting neurons ascend along the gut, DiI-labeled preparations were immunostained for calretinin. In the six preparations tested, only one neuron in one preparation was retrogradely labeled with DiI and expressed immunoreactivity for calretinin; the remainder of the DiI-labeled neurons were calretininnegative (Fig. 3B).

\section{A subset of projection neurons is calbindin-positive}

The myenteric neurons that express immunoreactivity for calbindin have been identified as intrinsic sensory neurons in the guinea pig small intestine (Costa et al., 1992; Song et al., 1994; Kunze et al., 1995). These neurons have projections to the mucosa and respond to luminal stimuli. We found that a subset of DiI-positive projection neurons were calbindin-IR (Fig. 4A-D). Computer-generated maps were produced to determine the distributions of retrogradely labeled cells that were calbindinpositive (Fig. $4 E$ ). In six preparations, the mean number of DiI-labeled-calbindin-IR neurons was $27 \pm 3.4$ per preparation (range, 12-37). The average number of retrogradely labeled neurons in these same preparations was 131.2 cells (range, 79-175); therefore, the proportion of double-labeled neurons for DiI and calbindin-IR was $20.6 \pm 2.1 \%$ (range, 15.1-28.2\%). The mean distance of the DiI-labeled-calbindin-IR cells from the SO was $2477.95 \pm 140.3 \mu \mathrm{m}$.

\section{Calbindin-IR nerve fibers in the SO degenerate when the SO is maintained in organ culture}

The results described above demonstrate that calbindin-IR neurons send processes to the SO, but it is not clear whether SO neurons are a target of this projection. In the guinea pig, SO ganglia are richly innervated by calbindin-IR nerve fibers, but these ganglia contain few, if any, calbindin-IR neurons (Fig. 5A) (Wells and Mawe, 1993). Because nearby myenteric ganglia of the duodenum contain a relatively high proportion $(25 \%)$ of calbindin-positive neurons (Fig. 5B), they represent a likely source of calbindin-IR fibers in SO ganglia. Two different organ culture preparations were used to test whether calbindin-IR fibers in SO ganglia originate in the myenteric plexus of the duodenum. Whole-mount preparations consisting of either an isolated SO $(n=6)$, or the SO with the duodenum attached $(n=6)$, were maintained in organotypic culture for $72 \mathrm{hr}$ and then immunostained for calbindin. In SO preparations cultured alone, occasional calbindin-IR neurons were present, but there was a dramatic reduction in calbindin-IR nerve fibers in the ganglia (Fig. $5 C$ ). When the SO was cultured with the duodenum attached, calbindin-IR fibers were present in the SO ganglia and could be seen passing into the SO from the duodenum along interganglionic connectives (Fig. 5D). These data support the concept that a duodenum-SO projection includes calbindin-IR nerve fibers and that these axons terminate in SO ganglia.

\section{CCK depolarizes duodenum-SO projection neurons}

To determine whether duodenum-SO projection neurons express CCK receptors and, therefore, could be capable of detecting the release of mucosal CCK, DiI-labeled cells were impaled with intracellular recording electrodes, and CCK was applied by pressure microejection or by superfusion. For these experiments, attempts were made to selectively impale large DiI-filled neurons containing several processes. This active selection of neurons was made in an attempt to record from neurons likely to be Dogiel type II in morphology and, thus, also likely to be intrinsic sensory neurons in this system. To ensure that the recordings were actually made from the targeted DiI-filled cell, neurobiotin was iontophoretically injected from the recording electrode for subsequent localization. In some cases $(n=2)$, a neuron adjacent to the DiI-filled cell was inadvertently impaled. Nine DiI-labeled cells from seven different preparations were successfully impaled, as confirmed by the presence of peroxidase reaction product (from neurobiotin coupled to avidin peroxidase) in DiI-labeled cells (Fig. 6B). Seven of these cells had numerous long processes, and two cells had a single long process and several short lamellar processes. These morphologies are characteristic of Dogiel type II and Dogiel type I cells, respectively, see (Furness and Costa, 1987). All of the impaled neurons responded to application of CCK with a prolonged depolarization (Fig. 6D). The depolarization had an amplitude of $11.4 \pm 5.0 \mathrm{mV}$ (range, $4-20 \mathrm{mV}$ ) and a duration of $68.9 \pm 15.3 \mathrm{sec}$ (range, 25-145 sec). During this depolarization, some cells generated bursts of action potentials. 

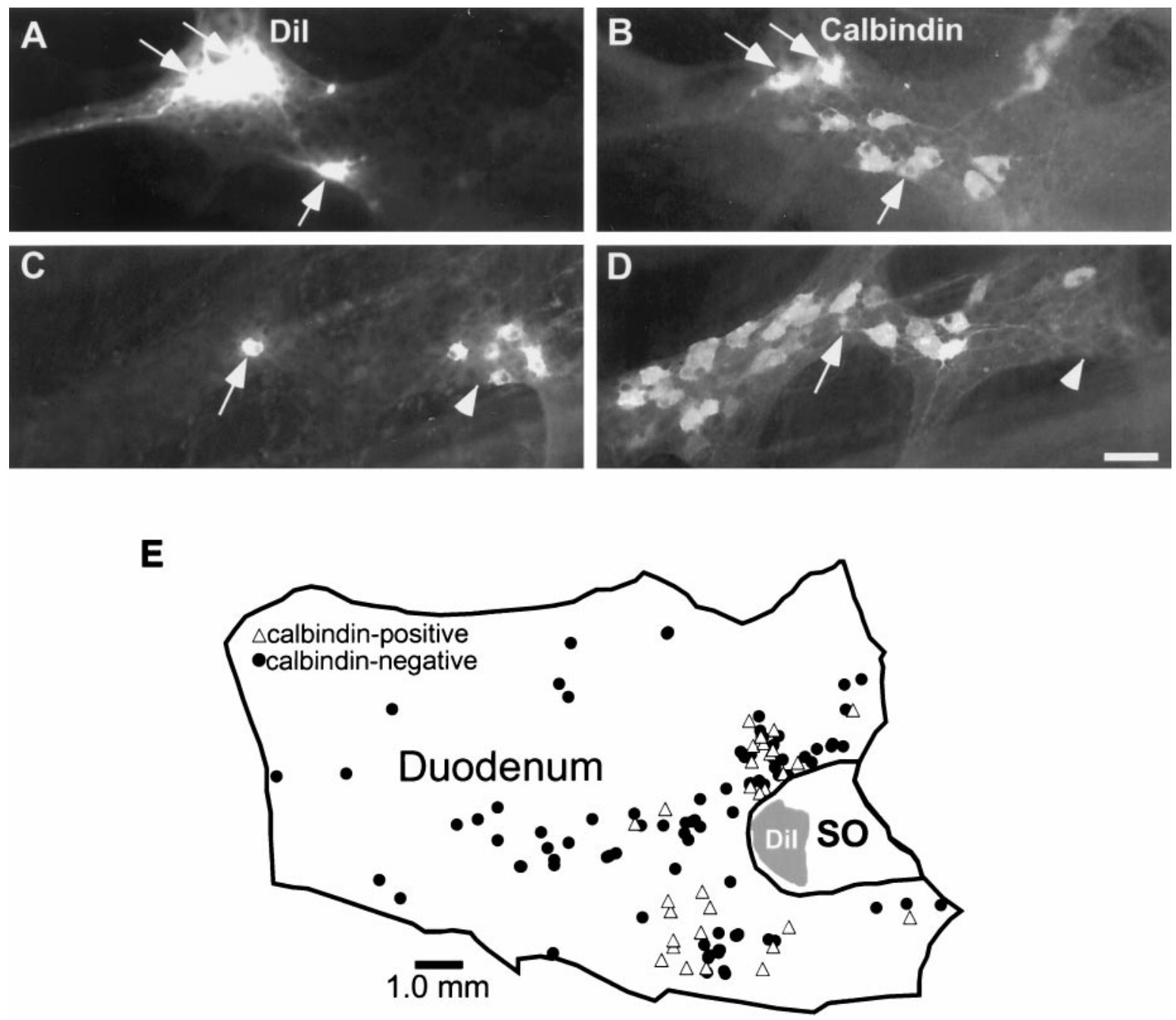

Figure 4. A subpopulation of the duodenum-SO-projecting neurons is calbindin-IR. $A-D$ demonstrate matching photomicrographs of two fields demonstrating DiI $(A, C)$ and calbindin immunoreactivity-labeled with FITC $(B, D)$. DiI-labeled neurons that are also calbindin-IR are indicated by arrows. A cluster of DiI-labeled neurons that are calbindin-negative is indicated by arrowheads. E, A computer-generated map of a representative preparation demonstrating the locations of DiI-labeled neurons in the duodenum that were calbindin-positive (triangles) or calbindin-negative (dots). In this preparation, 30 neurons were calbindin-positive of a total of 124 DiI-labeled neurons. Scale bar: $A-D, 50 \mu \mathrm{m}$.

\section{DISCUSSION}

This study was designed to test the hypothesis that a neural link exists between the duodenum and the SO and that this link could form the basis of an information pathway that signals the release of mucosal CCK. These results provide evidence that cholinergic neurons in the duodenal myenteric plexus project to the SO. Some of these neurons are primary afferent neurons, as defined by their calbindin immunoreactivity, and these neurons project to $\mathrm{SO}$ ganglia. The myenteric neurons that express immunoreactivity for calbindin have been identified as intrinsic sensory neurons in the guinea pig small intestine (Costa et al., 1992; Song et al., 1994; Kunze et al., 1995) and have been shown to send processes to the mucosa (Furness et al., 1990; Song et al., 1994; Clerc et al., 1998b). Furthermore, duodenal neurons that project to the SO are sensitive to CCK. These results demonstrate that SO tone could be regulated by neural inputs from the duodenum and that this circuitry could account for changes in SO resistance that follow postprandial release of CCK from the duodenal mucosa.

The results provided here, demonstrating the existence of neural projection to the SO from the duodenum, are consistent with other evidence supporting such a projection. In the current study, application of DiI to the guinea pig SO resulted in retrograde labeling of neurons in the myenteric plexus of the duodenum. Retrograde labeling of duodenal neurons from the SO has also been reported in the Australian possum after in vivo injections of DiI into the wall of the SO (Padbury et al., 1993). Furthermore, in physiological experiments also performed in the Australian possum, electrical field stimulation or mechanical distention of the duodenum caused changes in SO tone that were blocked when the junction between SO and duodenum was crushed (Saccone et al., 1994). Wells and Mawe (1993) also proposed that projections between the duodenum and SO exist in the guinea pig on the basis of calbindin immunoreactivity in SO ganglia. Ganglia in the SO contain a rich network of calbindin-IR fibers but very few calbindin-positive neurons. On the other hand, ganglia in the adjacent duodenum contain many calbindin-IR cell bodies, and Wells and Mawe (1993) have speculated that calbindin-positive nerves fibers in SO ganglia may arise in duodenal ganglia.

Recent evidence suggests that myenteric ganglia of the guinea 

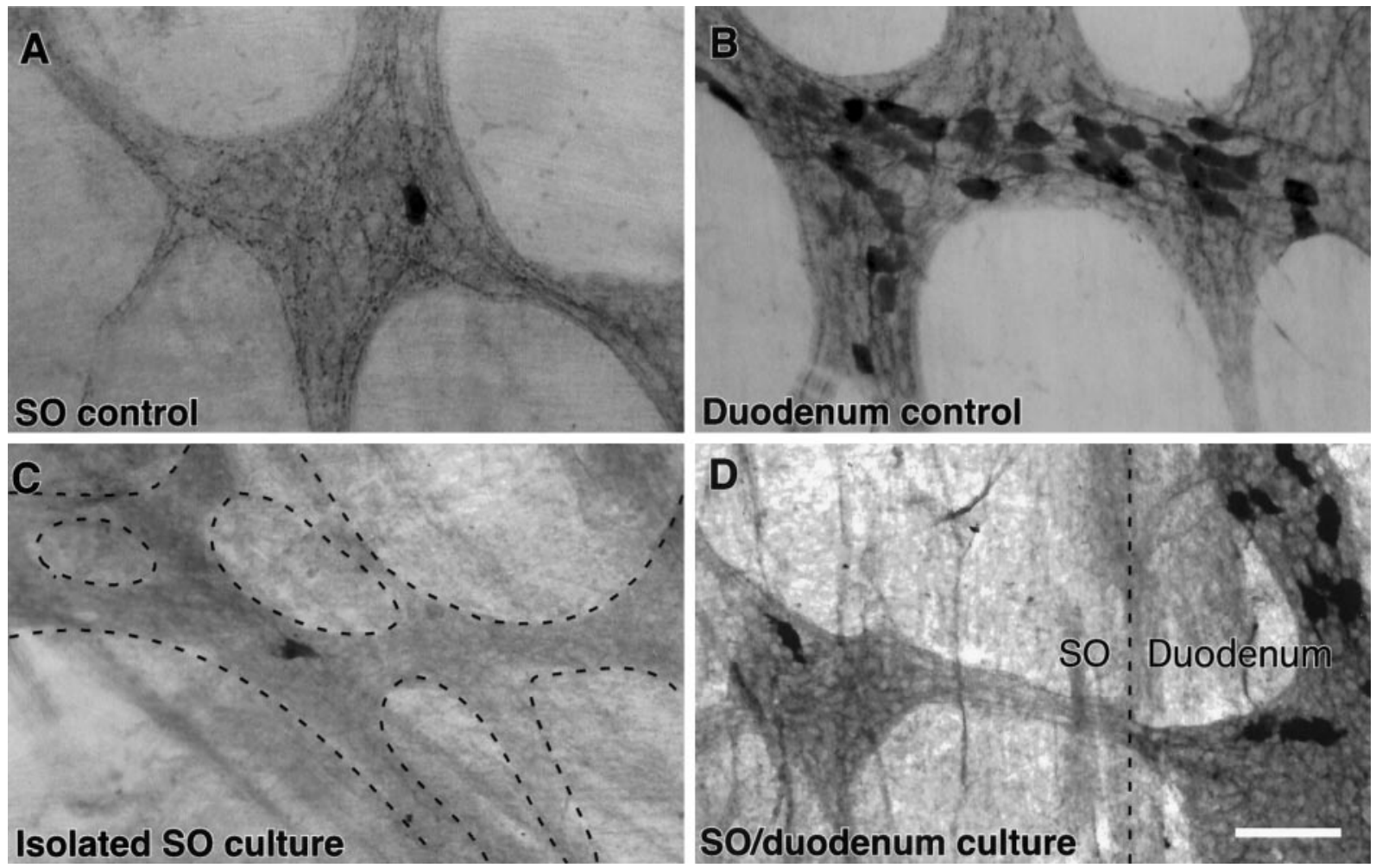

Figure 5. Calbindin-positive fibers in the SO degenerated when the SO was maintained in organ culture. $A, B$, Control preparations of the SO $(A)$ and duodenal $(B)$ ganglia that were fixed immediately and immunostained for calbindin and visualized with a DAB reaction product. Note the large number of calbindin-positive nerve fibers in the SO ganglia, relative to the single calbindin-positive neuron and the large number of calbindin-positive neurons in the duodenal ganglion. $C$, Photomicrograph of a region of the ganglionated plexus in the SO (dashed lines) that was immunostained for calbindin after the SO had been cultured in isolation for $72 \mathrm{hr}$. Note the absence of calbindin-positive nerve fibers. $D$, Photomicrograph of the interface between the $\mathrm{SO}$ and the duodenum (dashed line) in a preparation that was immunostained for calbindin after the SO had been cultured for $72 \mathrm{hr}$ with the duodenum intact. Calbindin-immunoreactive nerve fibers can be seen in the interganglionic nerve bundle that passes between the duodenal and SO ganglia. Scale bar, $50 \mu \mathrm{m}$.

pig duodenum consist of several subtypes of neurons, based on projections, chemical coding patterns, cell shapes, and electrical properties (Clerc et al., 1998a,b). In general, these classes of neurons are quite similar to those of the guinea pig ileum, which have been extensively characterized (Furness and Costa, 1987; Costa and Brookes, 1994; Gershon et al., 1994). Throughout the duodenum and small intestine, the vast majority of neurons are immunoreactive for ChAT along with other excitatory transmitters such as substance $\mathrm{P}$, or they are nitrergic. Neurons in the duodenum of the guinea pig that project to the SO are likely to be cholinergic because they are IR for ChAT but not NOS. This indicates that the neural input to the SO from the duodenum is likely to be excitatory and may represent a source of the nicotinic fast synaptic inputs that most SO neurons receive (Wells and Mawe, 1993).

In the guinea pig small intestine and duodenum, interneurons with ascending projections express ChAT-IR, and they also express IR for the calcium-binding protein calretinin (Brookes et al., 1991a; Clerc et al., 1998b). Although the duodenal neurons that were retrogradely labeled with DiI expressed ChAT-IR, and many projected to the SO in an ascending direction, they were not immunoreactive for calretinin. This indicates that the duodenum-SO circuit comprises a set or sets of myenteric neurons that are distinct from the ascending neurons that are thought to be involved in the oral contraction associated with peristaltic activity.

A subset of the duodenum-SO projection neurons are likely to be intrinsic sensory neurons of the myenteric plexus. In this study, $\sim 20 \%$ of the duodenal neurons that were retrogradely labeled from the SO were immunoreactive for calbindin. During the past decade, the myenteric neurons that express IR for calbindin have been identified as intrinsic sensory neurons of the guinea pig small intestine (Song et al., 1994; Kunze et al., 1995). Multidisciplinary studies have demonstrated that these neurons correspond to the population of neurons that have been categorized electrically as type 2/AH cells and morphologically as Dogiel type II cells. Dogiel type II cells have several long processes emanating from the soma that pass through and appear to synapse in numerous ganglia. Processes of these neurons, at least the majority that are calbindin-positive, also extend to the mucosa. Song et al. (1994) demonstrated that all calbindin-IR neurons project to the mucosa of the small intestine in which calbindin-positive fibers exist in each villus. Furthermore, Furness et al. (1990) showed that the myenteric plexus is the principal source of calbindin-IR nerve fibers in the mucosa, because calbindin immunoreactivity was eliminated from the mucosal layer after local lesions of the myenteric plexus. More recently, Clerc et al. (1998b) have shown that calbindin-IR neurons in the duodenum also have projections that pass to the mucosa. Therefore, the calbindin-positive DiIlabeled neurons would be capable of serving as primary sensory neurons in a duodenum-SO neural reflex circuit, as well as acting as projection neurons.

From the retrograde-labeling studies alone, it is not possible to determine specifically where the duodenum-SO projection neu- 

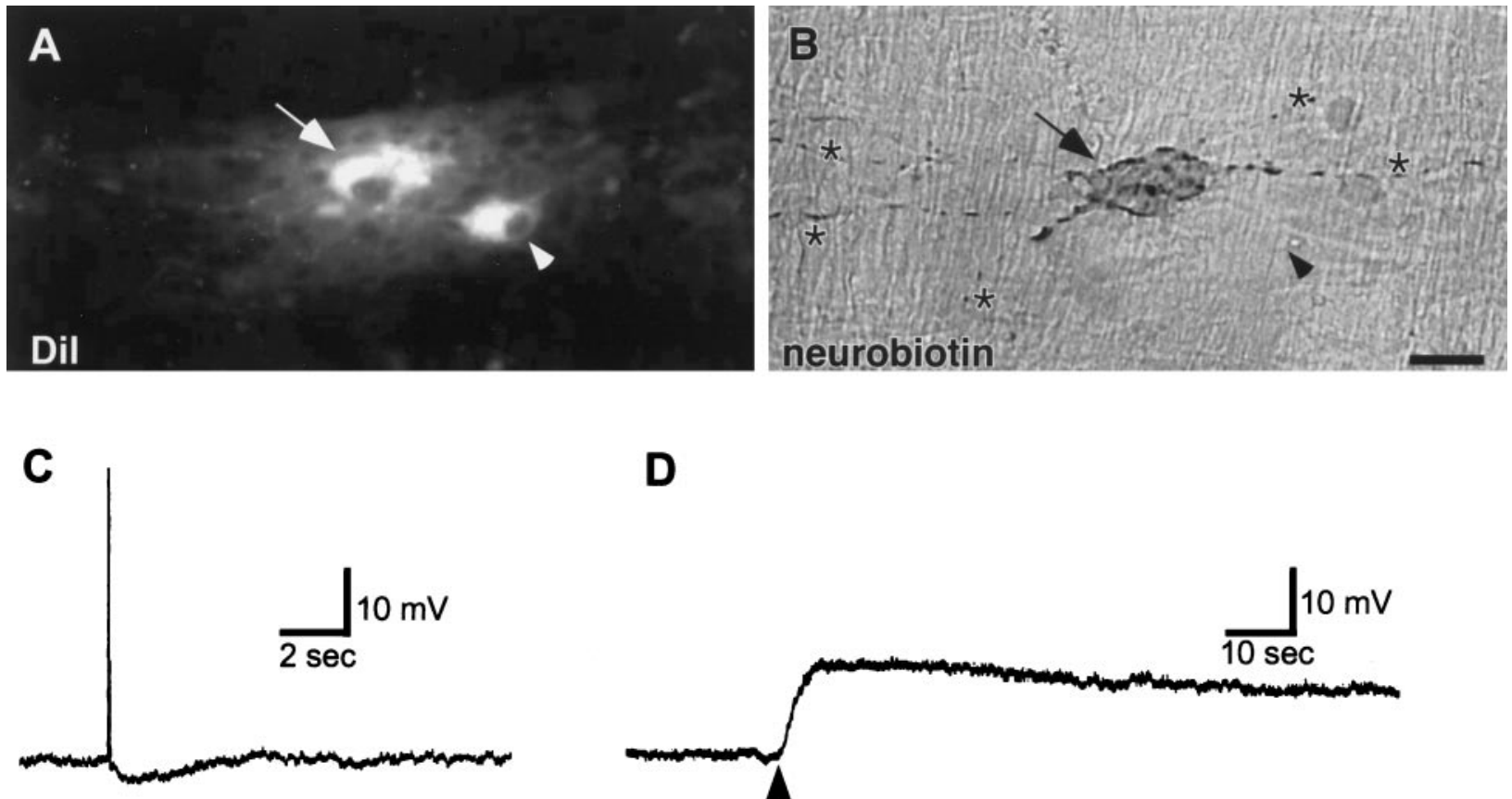

D

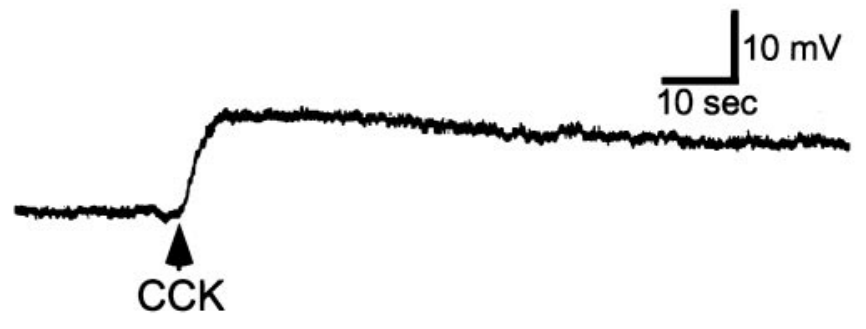

Figure 6. Neurons that project from the duodenum to the SO are sensitive to CCK. $A, B$, Photomicrographs of the same field demonstrating DiI fluorescence $(A)$ and neurobiotin-labeled with a DAB reaction product $(B)$. The neuron that was impaled with an intracellular microelectrode, filled with neurobiotin, and exposed to CCK is indicated by the arrows. The location of a nearby neuron that was retrogradely labeled with DiI but not studied electrophysiologically is indicated with arrowheads. The numerous processes of the neurobiotin-filled neuron, which is a Dogiel type II cell, are indicated by asterisks. $C, D$, Electrical recordings of the neurobiotin-filled neuron shown in $B$. $C$, Electrical traces demonstrating that the action potential of this neuron was followed by a prolonged afterhyperpolarization, which is characteristic of type $2 / \mathrm{AH}$ neurons. $D$, Pressure microejection of CCK ( 2 sec) resulted in a prolonged depolarization. Scale bar: $A, B, 25 \mu \mathrm{m}$.

rons terminate in the SO. Because DiI diff used over a large area at the application site within the SO, axon terminals that took up the dye could have been in the ganglionated plexus or in muscle. The results of SO organ culture studies indicate that calbindin-IR duodenal neurons projecting to SO terminate in SO ganglia, but it is also possible that some of the retrogradely labeled neurons project through, and not necessarily to, the SO. Control SO ganglia and SO ganglia maintained in organ culture with the duodenum intact contained an abundance of calbindin-IR varicose nerve fibers, but the ganglionated plexuses of SO preparations organ cultured in isolation were almost devoid of calbindin-IR fibers. Thus, calbindin-IR neurons are capable of delivering a monosynaptic reflex signal from the mucosa of the duodenum to the ganglia, and possibly to motor neurons, of the SO.

The question remains, what is the purpose of a neural input to the SO from the duodenum? The functions of the SO are to regulate the flow of bile and pancreatic juices into the duodenum and to prevent the diversion of intestinal contents into the biliary ducts. The SO remains contracted between meals, thus restricting the flow of bile into the duodenum and routing bile to the gallbladder for storage and concentration. Postprandially, CCK released from the duodenal mucosa causes the SO musculature to relax or pump, depending on the species, allowing bile to flow from the gallbladder into the duodenum. Several studies in various species have shown that the actions of CCK on the SO are sensitive to neural blockade (Behar and Biancani, 1987; Vogalis et al., 1989; Hanyu et al., 1990). Furthermore, CCK can cause the release of several neuroactive compounds in the SO, including acetylcholine (Harada et al., 1986), vasoactive intestinal peptide (Wiley et al., 1988; Dahlstrand et al., 1990), and nitric oxide
(Pauletzki et al., 1993). However, it is unlikely that CCK alters SO ganglionic output through a direct hormonal action on SO neurons. We have recently shown that, although SO neurons express CCK receptors, the concentrations of CCK necessary to evoke a detectable response are at least 100-fold higher than the concentrations of CCK present in the serum after a meal (Gokin et al., 1997).

Data from the present study support the hypothesis that a neural circuit could mediate the actions of mucosal CCK on SO tone. Mucosal CCK could have paracrine stimulatory effects on the calbindin-IR, duodenum-SO projection neurons. As mentioned above, calbindin-IR myenteric neurons have projections to the mucosa, and thus are morphologically suited for such a role. Furthermore, these neurons express CCK receptors, because DiIlabeled Dogiel type II cells were depolarized by CCK. Others have also shown that CCK can depolarize type 2/AH cells of the duodenum (Mutabagani et al., 1993) and the ileum (Nemeth et al., 1985; Schutte et al., 1997), which have a Dogiel type II morphology. It is interesting though, that all of the duodenumSO-projecting neurons that tested in the current study responded to CCK, whereas in the previous studies, CCK elicited responses in only a subset of myenteric neurons that were randomly impaled.

The function of the remaining $80 \%$ of the duodenum-SO projection neurons, which are ChAT-positive and calbindinnegative, may be related to a coordination of SO tone and duodenal motor activity. Local duodenal contractions are likely to be associated with SO contractions to prevent the movement of luminal contents into the bile duct. A neural circuit between the myenteric plexus of the duodenum and the ganglia of the SO may 
aid in coordinating these motor responses. Another potential role for the duodenum-SO projection is to coordinate SO relaxations with the migrating myoelectric complex. These events, which occur every few hours and involve a peristaltic wave that travels the length of the gut, are associated with a coordinated discharge of bile into the duodenum. It is possible that duodenum-SOprojecting neurons, as well as the duodenum-gallbladderprojecting neurons that have been demonstrated (Mawe and Gershon, 1989; Padbury et al., 1993), could regulate the biliary response with the migrating myoelectric complex.

In summary, these data demonstrate that a projection from the duodenal myenteric plexus to the SO exists in the guinea pig. Neurons that contribute to this projection are cholinergic, and a subset of these neurons are primary sensory neurons that have the capacity to detect CCK. This duodenum-SO circuit could be responsible for postprandial changes in SO tone that facilitate the delivery of bile and pancreatic juices into the duodenum. Furthermore, this neural connection between the duodenum and the SO may allow for the coordination of motor activities between these two regions of the gut.

\section{REFERENCES}

Bayliss WM, Starling EH (1899) The movements and innervation of the small intestine. J Physiol (Lond) 24:99-143.

Behar J, Biancani P (1987) Pharmacologic characterization of excitatory and inhibitory cholecystokinin receptors of the cat gallbladder and sphincter of Oddi. Gastroenterology 92:764-770.

Brookes SJH, Steele PA, Costa M (1991a) Calretinin immunoreactivity in cholinergic motor neurones, interneurones and vasomotor neurones in the guinea pig small intestine. Cell Tissue Res 263:471-481.

Brookes SJH, Steele PA, Costa M (1991b) Identification and immunohistochemistry of cholinergic and non-cholinergic circular muscle motor neurons in the guinea-pig small intestine. Neuroscience 42:863-878.

Clerc N, Furness JB, Bornstein JC, Kunze WAA (1998a) Correlation of electrophysiological and morphological characteristics of myenteric neurons of the duodenum in the guinea-pig. Neuroscience 82:899-914.

Clerc N, Furness JB, Li ZS, Bornstein JC, Kunze WAA (1998b) Morphological and immunohistochemical identification of neurons and their targets in the guinea-pig duodenum, in press.

Costa M, Brookes SJH (1994) The enteric nervous system. Am J Gastroenterol 89:S129-S137.

Costa M, Brooks S, Waterman S, Mayo R (1992) Enteric neuronal circuitry and transmitters controlling intestinal motor function. In: Advances in the innervation of the gastrointestinal tract (Holle GE, Wood JD, eds), pp 115-121. Amsterdam: Elsevier Science.

Dahlstrand C, Dahlström A, Theodorsson E, Rehfeld J, Ahlman H (1990) Is the CCK-8 induced relaxation of the feline sphincter of Oddi mediated by VIP neurons? J Auton Nerv Syst 31:75-84.

Furness JB, Costa M (1987) The enteric nervous system. New York: Churchill-Livingston.

Furness JB, Trussell DC, Pompola S, Bornstein JC, Smith TK (1990) Calbindin neurons of the guinea-pig small intestine: quantitative analysis of their numbers and projections. Cell Tissue Res 260:261-272.

Gershon MD, Kirchgessner AL, Wade PR (1994) Functional anatomy of the enteric nervous system. In: Physiology of the gastrointestinal tract (Johnson LR, ed), pp 381-422. New York: Raven.
Gokin AP, Hillsley K, Mawe GM (1997) Cholecystokinin (CCK) depolarizes guinea pig sphincter of Oddi neurons by activating CCK-A receptors. Am J Physiol 272:G1365-G1374.

Hanyu N, Dodds WJ, Layman RD, Hogan WJ (1990) Cholecystokinininduced contraction of opossum sphincter of Oddi. Dig Dis Sci 35:567-576.

Harada T, Katsuragi T, Furukawa T (1986) Release of acetylcholine mediated by cholecystokinin receptor from the guinea pig sphincter of Oddi. J Pharmacol Exp Ther 239:554-558.

Honig MG, Hume RI (1989) DiI and DiO: versatile fluorescent dyes for neuronal labelling and pathway tracing. Trends Neurosci 12:333-341.

Kunze WAA, Bornstein JC, Furness JB (1995) Identification of sensory nerve cells in a peripheral organ (the intestine) of a mammal. Neuroscience $66: 1-4$.

Mawe GM (1995) Prevertebral, pancreatic and gallbladder ganglia: nonenteric ganglia that are involved in gastrointestinal function. In: Autonomic ganglia (McLachlan EM, ed), pp 397-444. Luxembourg: Harwood Academic.

Mawe GM, Gershon MD (1989) Structure, afferent innervation, and transmitter content of ganglia of the guinea pig gallbladder: relationship to the enteric nervous system. J Comp Neurol 283:374-390.

Mutabagani KH, Tamura K, Wood JD (1993) Actions of cholecystokinin (CCK) on morphologically identified neurons in the myenteric plexus of guinea-pig duodenum. Gastroenterology [Suppl] 104:A252.

Nemeth PR, Zafirov DH, Wood JD (1985) Effects of cholecystokinin, caerulein and pentagastrin on electrical behaviour of myenteric neurones. Eur J Pharmacol 116:263-269.

Padbury RTA, Furness JB, Baker RA, Toouli J, Messenger JP (1993) Projections of nerve cells from the duodenum to the sphincter of Oddi and gallbladder of the Australian possum. Gastroenterology 104:130-136.

Pauletzki JG, Sharkey KA, Davison JS, Bomzon A, Shaffer EA (1993) Involvement of L-arginine-nitric oxide pathways in neural relaxation of the sphincter of Oddi. Eur J Pharmacol 232:263-270.

Saccone GTP, Harvey JR, Baker RA, Toouli J (1994) Intramural neural pathways between the duodenum and sphincter of Oddi in the Australian brush-tailed possum in vivo. J Physiol (Lond) 481:447-456.

Schutte IW, Akkermans LM, Kroese AB (1997) CCKA and CCKB receptor subtypes both mediate the effects of CCK-8 on myenteric neurons in the guinea-pig ileum. J Auton Nerv Syst 67:51-9.

Song Z, Brookes SJH, Steele PA, Costa M (1992) Projections and pathways of submucous neurons to the mucosa of the guinea-pig small intestine. Cell Tissue Res 269:87-98.

Song Z-M, Brookes SHJ, Costa M (1994) All calbindin-immunoreactive myenteric neurons project to the mucosa of guinea-pig small intestine. Neurosci Lett 180:219-222.

Vogalis F, Bywater RAR, Taylor GS (1989) Propulsive activity of the isolated choledochoduodenal junction of the guinea pig. J Gastrointest Motil 1:115-121.

Wells DG, Mawe GM (1993) Physiological and morphological properties of neurons in the sphincter of Oddi region of the guinea pig. Am J Physiol 265:G258-G269.

Wells DG, Talmage EK, Mawe GM (1995) Immunohistochemical Identification of Neurons in Ganglia of the Guinea Pig Sphincter of Oddi. J Comp Neurol 352:106-116.

Wiley JW, O'Dorisio TM, Owyang C (1988) Vasoactive intestinal polypeptide mediates cholecystokinin-induced relaxation of the sphincter of Oddi. J Clin Invest 81:1920-1924. 